\title{
Physician Responsiveness to Positive Blood Culture Results at the Minneapolis Veterans Affairs Hospital-Is Anyone Paying Attention?
}

\author{
Shaun Heimbichner Johnson; and Steven James Waisbren, MD, PhD
}

Background: Patients presenting with suspected infections are typically placed on empiric broad-spectrum antibiotics. With mounting evidence supporting the efficacy of using the narrowest spectrum of antimicrobial therapy to cover the suspected pathogen, current guidelines recommend decreasing the breadth of coverage in response to culture results both in relation to microbe identification and antibiotic sensitivity.

Methods: A retrospective chart review of electronic health records at the Minneapolis Veterans Affairs Medical Center (VAMC) in Minnesota was performed for 208 positive blood cultures with antibiotic spectrum analysis from July 1, 2015 to June 30,2016 . The time of reporting for pathogen identification and subsequent pathogen susceptibilities were compared to the time at which any alterations to antibiotic coverage were made. The breadth of antibiotic coverage was recorded using a nonlinear spectrum score. The use of this score allowed for the reliable classification of antibiotic adjustments as either deescalation, escalation, or no change.

Results: The percentage of cases deescalated was higher in response to physician (house staff or attending physician) notification of pathogen susceptibility information when compared with a response to pathogen identification alone (33.2\% vs $22.6 \%$ ). Empiric antibiotics were not altered within 24 hours in response to pathogen identification in $70.7 \%$ of cases and were not altered within 24 hours in response to pathogen sen- sitivity determination in $58.6 \%$ of cases. However, when considering the time frame from when empiric antibiotics were started to 24 hours after notification of susceptibility information, $49.5 \%$ of cases were deescalated and $41.5 \%$ of cases had no net change in the antibiotic spectrum score. The magnitude of deescalations were notably larger than escalations. The mean (SD) time to deescalation of antibiotic coverage was shorter $(P=.049)$ in response to pathogen identification at 8 (7.4) hours compared with sensitivity information at 10.4 (7) hours, but may not be clinically relevant.

Conclusion: Health care providers at the Minneapolis VAMC appear to be using positive blood culture results in a timely fashion consistent with best practices. Because empirically initiated antibiotics typically are broad in spectrum, the magnitude of deescalations were notably larger than escalations. Adherence to these standards may be a reflection of the infectious disease staff oversight of antibiotic administration. Furthermore, the systems outlined in this quality improvement study may be replicated at other VAMCs across the country by either in-house infectious disease staff or through remote monitoring of the electronic health record by other infectious disease experts at a more centralized VAMC. Widespread adoption throughout the Veterans Health Administration may result in improved antibiotic resistance profiles and better clinical outcomes for our nation's veterans.

\begin{abstract}
Shaun Johnson is a
Medical Student at Case Western Reserve University School of Medicine in Cleveland, Ohio. Steven Waisbren is a Surgeon and Assistant Service Chief at the Minneapolis Veterans Affairs Health Care System in Minnesota and an Assistant Professor of Surgery at the University of Minnesota. Correspondence:

Steven Waisbren
\end{abstract}

(steven.waisbren@va.gov)

Fed Pract. 2021;38(3):128-135. doi:10.12788/fp.0101
T he US Department of Veterans Affairs (VA) is the largest health care organization in the US, staffing more than 1,200 facilities and servicing about 9 million veterans. ${ }^{1}$ Identifying VA practices that promote effective health care delivery has the potential to impact thousands of patients every day. The Surgical service at the Minneapolis VA Medical Center (MVAMC) in Minnesota often questioned colleagues whether many of the ordered tests, including blood cultures for patients with suspected infections, were clinically necessary. Despite recommendations for utilizing culture-driven results in choosing appropriate antimicrobials, it was debated whether these additional tests were simply drawn and ignored resulting only in increased costs and venipuncture discomfort for the patient. Thus, the purpose of this quality improvement study was to determine whether positive blood culture results actually influence clinical management at MVAMC.

\section{BACKGROUND}

Accepted best practice when responding to positive blood culture results entails empiric treatment with broad-spectrum antibiotics that subsequently narrows in breadth of coverage once the pathogen has been identified. ${ }^{2-4}$ This strategy has been labeled deescalation. Despite the acceptance of these standards, surveys of clinician attitudes towards antibiotics showed that 90\% of physicians and residents stated they wanted more education on antimicrobials and $80 \%$ desired better schooling on antibiotic choices. ${ }^{5,6}$ Additionally, in an online survey $18 \%$ of 402 inpatient and emergency department providers, including residents, fellows, intensive care unit (ICU) and emergency department attending physicians, hospitalists, physician assistants, and nurse 
practitioners, described a lack of confidence when deescalating antibiotic therapy and $45 \%$ reported that they had received training on antimicrobial prescribing that was not fully adequate. ${ }^{7}$

These surveys hint at a potential gap in provider education or confidence, which may serve as a barrier to ideal care, further confounding other individualized considerations taken into account when deescalating care. These considerations include patient renal toxicity profiles, the potential for missed pathogens not identified in culture results, unknown sources of infection, and the mindset of many providers to remain on broad therapy if the patient's condition is improving. ${ }^{8-10}$ A specific barrier to deescalation within the VA is the variance in antimicrobial stewardship practices between facilities. In a recent widespread survey of VA practices, Chou and colleagues identified that only 29 of $130(22.3 \%)$ responding facilities had a formal policy to establish an antimicrobial stewardship program. ${ }^{11}$

Overcoming these barriers to deescalation through effective stewardship practices can help to promote improved clinical outcomes. Most studies have demonstrated that outcomes of deescalation strategies have equivalent or improved mortality and equivalent or even decreased length of ICU stay. ${ }^{12-26}$ Although a 2014 study by Leone and colleagues reported longer overall ICU stay in deescalation treatment groups with equivalent mortality outcomes, newer data do not support these findings. ${ }^{16,20,22}$

Furthermore, antibiotics can be expensive. Deescalation, particularly in response to positive blood culture results, has been associated with reduced antibiotic cost due to both a decrease in overall antibiotic usage and the utilization of less expensive choices. ${ }^{22,24,26,27}$ The findings of these individual studies were corroborated in 2013 by a meta-analysis, including 89 additional studies. ${ }^{28}$ Besides the direct costs of the drugs, the development of regional antibiotic resistance has been labeled as one of the most pressing concerns in public health, and major initiatives have been undertaken to stem its spread. ${ }^{29,30}$ The majority of clinicians believe that deescalation of antibiotics would reduce antibiotic resistance. Thus, deescalation is widely cited as one of the primary goals in the management of resistance development. ${ }^{5,24,26,28,31,32}$

Due to the proposed benefits and challenges of implementation, MVAMC instituted a program where the electronic health records (EHR) for all patients with positive blood culture results were reviewed by the on-call infectious disease attending physician to advise the primary care team on antibiotic administration. The MVAMC system for notification of positive blood culture results has 2 components. The first is phone notification to the on-call resident when the positive result of the pathogen identification is noted by the microbiology laboratory staff. Notably, this protocol of phone notification is only performed when identifying the pathogen and not for the subsequent sensitivity profile. The second component occurs each morning when the on-call infectious disease attending physician reviews all positive blood culture results and the current therapy. If the infectious disease attending physician feels some alterations in management are warranted, the physician calls the primary service. Additionally, the primary service may always request a formal consult with the infectious disease team. This quality improvement study was initiated to examine the success of this deescalation/stewardship program to determine whether positive blood culture results influenced clinical management.

\section{METHODS}

From July 1, 2015 to June 30, 2016, 212 positive blood cultures at the MVAMC were analyzed. Four cases that did not have an antibiotic spectrum score were excluded, leaving 208 cases reviewed. Duplicate blood cultures were excluded from analysis. The microbiology laboratory used the BD Bactec automated blood culture system using the Plus aerobic and Lytic anaerobic media (Becton, Dickinson and Company).

Antibiotic alterations in response to culture results were classified as either deescalation or escalation, using a spectrum score developed by Madaras-Kelly and colleagues. ${ }^{33}$ These investigators performed a 3-round modified Delphi survey of infectious disease staff of physicians and pharmacists. The resulting consensus spectrum score for each respective antibiotic reflected the 
TABLE 1 Classification of Culture Results $(\mathrm{N}=208)$

\begin{tabular}{lcc} 
Groups & $\begin{array}{c}\text { Within 24 h of Pathogen } \\
\text { Identification, No (\%) }\end{array}$ & $\begin{array}{c}\text { Within 24 h of Antibiotic } \\
\text { Sensitivities, No. (\%) }\end{array}$ \\
\hline Deescalated & $47(22.6)$ & $69(33.2)$ \\
\hline Escalated & $14(6.7)$ & $17(8.2)$ \\
\hline No antibiotic alteration & $147(70.7)$ & $122(58.6)$ \\
\hline Total & $208(100)$ & $208(100)$
\end{tabular}

relative susceptibilities of various pathogens to antibiotics and the intrinsic resistance of the pathogens. It is a nonlinear scale from 0 to 60 with a score of 0 indicating no antibacterial activity and a score of 60 indicating complete coverage of all critically identified pathogens. For example, a narrow-spectrum antibiotic such as metronidazole received a spectrum score of 4.0 and a broad-spectrum antibiotic such as piperacillin/tazobactam received a 42.3 score.

Any decrease in the spectrum score when antibiotics were changed was described as deescalation and an increase was labeled escalation. In cases where multiple antibiotics were used during empiric therapy, the cessation of $\geq 1$ antibiotics was classified as a deescalation while the addition of $\geq 1$ antibiotics was classified as an escalation.

Madaras-Kelly and colleagues calculated changes in spectrum score and compared them with Delphi participants' judgments on deescalation with 20 antibiotic regimen vignettes and with non-Delphi steward judgments on deescalation of 300 pneumonia regimen vignettes. Antibiotic spectrum scores were assigned a value for the width of empiric treatment that was compared with the antibiotic spectrum score value derived through antibiotic changes made based on culture results. In the Madaras-Kelly cases, the change in breadth of antibiotic coverage was in agreement with expert classification in $96 \%$ of these VA patient cases using VA infectious disease specialists. This margin was noted as being superior to the inter-rater variability between the individual infectious disease specialists.

\section{Data Recording and Analysis}

Charts for review were flagged based on positive blood culture results from the mi- crobiology laboratory. EHRs were manually reviewed to determine when antibiotics were started/stopped and when a member of the primary care team, usually a resident, was notified of culture results as documented by the microbiology laboratory personnel. Any alteration in antibiotics that fit the criteria of deescalation or escalation that occurred within 24 hours of notification of either critical laboratory value was recorded. The identity of infectious pathogens and the primary site of infection were not recorded as these data were not within the scope of the purpose of this study. We did not control for possible contaminants within positive blood cultures.

There were 3 time frames considered when determining culture driven alterations to the antibiotic regimen. The first 2 were changes within the 24 hours after notification of either (1) pathogen identification or (2) pathogen sensitivity. These were defined as culture-driven alterations in response to those particular laboratory findings. The third-whole case time frame-spanned from pathogen identification to 24 hours after sensitivity information was recorded. In cases where $\geq 1$ antibiotic alteration was noted within a respective time frame, a classification of deescalation or escalation was still assigned. This was done by summing each change in spectrum score that occurred from antibiotic regimen alterations within the time frame, and classifying the net effect on the spectrum of coverage as either deescalation or escalation. Data were recorded in spreadsheet. RStudio 3.5.3 was used for statistical analysis.

\section{RESULTS}

Of 208 cases assigned a spectrum score, $47(22.6 \%)$ had the breadth of antibiotic coverage deescalated by the primary care team within 24 hours of pathogen identification with a mean (SD) physician response time of 8.0 (7.3) hours. Fourteen cases $(6.7 \%)$ had the breadth of antibiotic coverage escalated from pathogen identification with a mean (SD) response time of 8.0 (7.4) hours. When taken together, within 24 hours of pathogen identification from positive blood cultures 61 cases $(29.3 \%)$ had altered antibiotics, leaving $70.7 \%$ of cases un- 
altered (Tables 1 and 2). In this nonquantitative spectrum score method, deescalations typically involved larger changes in spectrum score than escalations.

Physician notification of pathogen sensitivities resulted in deescalation in 69 cases (33.2\%) within 24 hours, with a mean (SD) response time of 10.4 (7) hours. The mean time to deescalation in response to pathogen identification was significantly shorter than the mean time to deescalation in response to sensitivities $(P=.049)$. Broadening of coverage based on sensitivity information was reported for 17 cases (8.2\%) within 24 hours, with a mean (SD) response time of 7.6 (6) hours (Table 3 ). In response to pathogen sensitivity results from positive blood cultures, $58.6 \%$ of cases had no antibiotic alterations. Deescalations involved notably larger changes in spectrum score than escalations.

More than half $(58.6 \%)$ of cases resulted in an antibiotic alteration from empiric treatment when considering the time frame from empiric antibiotics to 24 hours after receiving sensitivity information. These were deemed the whole-case, culture-driven results. In addition to antibiotic alterations that occurred within 24 hours of either pathogen identification or sensitivity information, the wholecase category also considered antibiotic alterations that occurred more than 24 hours after pathogen identification was known and before sensitivity information was available, although this was rare. Some of these patients may have had their antibiotics altered twice, first after pathogen identification and later once sensitivities became available with the net effect recorded as the whole-case administration. Of those that had their antibiotics modified in response to laboratory results, by a ratio of $6.4: 1$, the change was a deescalation rather than an escalation.

\section{DISCUSSION}

The strategy of the infectious disease team at MVAMC is one of deescalation. One challenge of quantifying deescalation was to make a reliable and agreed-upon definition of just what deescalation entails. In 2003, the pharmaceutical company Merck was granted a trademark for the phrase "DeEscalation Therapy" under the international class code 41 for educational and entertainment services. This seemed to cor-
TABLE 2 Antibiotic Change Cases as a Result of Positive Blood Culture Results

\begin{tabular}{lc} 
Groups & $\begin{array}{c}\text { Cases Spanning Start of Empiric Antibiotics to } \\
\text { 24 } \mathbf{h} \text { After Antibiotic Sensitivities, No. (\%) }\end{array}$ \\
\hline Deescalated & $103(49.5)$ \\
\hline Escalated & $19(9.1)$ \\
\hline No change & $86(41.4)$ \\
\hline Total & $208(100)$
\end{tabular}

respond to marketing efforts for the antibiotic imipenem/cilastatin. Although the company trademarked the term, it was never defined. The usage of the phrase evolved from a reduction of the dosage of a specific antibiotic to a reduction in the number of antibiotics prescribed to that of monotherapy. The phrase continues to evolve and has now become associated with a change from combination therapy or broad-spectrum antibiotics to monotherapy, switching to an antibiotic that covers fewer pathogens, or even shortening the duration of antibiotic therapy. ${ }^{34}$ The trademark expired at about the same time the imipenem/ cilastatin patent expired. Notably, this drug had initially been marketed for use in empiric antibiotic therapy. ${ }^{35}$

\section{Barriers}

The goal of the stewardship program was not to see a narrowing of the antibiotic spectrum in all patients. Some diseases such as diverticulitis or diabetic foot infections are usually associated with multiple pathogens where relatively broad-spectrum antibiotics seem to be preferred. ${ }^{36,37}$ Heenen and colleagues reported that infectious disease specialists recommended deescalation in $<50 \%$ of cases they examined. ${ }^{38}$

Comparing different institutions' deescalation rates can be confusing due to varying definitions, differing patient populations, and health care provider behavior. Thus, the published rates of deescalation range widely from 10 to $70 \% .^{2,39,40}$ In addition to the varied definitions of deescalation, it is challenging to directly compare the rate of deescalation between studies due to institutional variation in empirical broad-spectrum antibiotic usage. A hospital that uses 
TABLE 3 Response Time of Antibiotic Changes in Response to Notification of Pathogen Identification and Sensitivity

\begin{tabular}{lcc} 
Cases & $\begin{array}{c}\text { Response Time for Antibiotic Alteration After } \\
\text { Notification of Pathogen Identification, } \\
\text { mean (SD), h }\end{array}$ & $\begin{array}{c}\text { Response Time for Antibiotic Alteration After } \\
\text { Notification of Pathogen Sensitivity, } \\
\text { mean (SD), h }\end{array}$ \\
\hline Deescalated & $8.0(7.3)$ & $10.4(7)$ \\
\hline Escalated & $8.0(7.4)$ & $7.6(6)$
\end{tabular}

broad-spectrum antibiotics at a higher rate than another has the potential to deescalate more often than one that has low rates of empirical broad-spectrum antibiotic use. Some studies use a conservative definition of deescalation such as narrowing the spectrum of coverage, while others use a more general definition, including both the narrowing of spectrum and/or the discontinuation of antibiotics from empirical therapy. ${ }^{41-45}$ The more specific and validated definition of deescalation used in this study may allow for standardized comparisons. Another unique feature of this study is that all positive blood cultures were followed, not only those of a particular disease.

One issue that comes up in all research performed within the VA is how applicable these results are to the general public. Nevertheless, the stewardship program as it is structured at the MVAMC could be applied to other non-VA institutions. We recognize, however, that some smaller hospitals may not have infectious diseases specialists on staff. Despite limited in-house staff, the same daily monitoring can be performed off-site through review of the EHR, thus making this a viable system to more remote VA locations.

While deescalation remains the standard of care, there are many complexities that explain low deescalation rates. Individual considerations that can cause physicians to continue the empirically initiated broadspectrum coverage include differing renal toxicities, suspecting additional pathogens beyond those documented in testing results, and differential Clostridium difficile risk. ${ }^{46,47}$ A major concern is the mind-set of many prescribers that streamlining to a different antibiotic or removing antibiotics while the patient is clinically improving on broad empiric therapy represents an unnecessary risk. ${ }^{48,49}$ These thoughts seem to stem from the old adage, "If it ain't broke, don't fix it."

Due to the challenges in defining deescalation, we elected to use a well-accepted and validated methodology of Madaras-Kelly. ${ }^{33}$ We recognize the limitations of the methodology, including somewhat differing opinions as to what may constitute breadth and narrowing among clinicians and the somewhat arbitrary assignment of numerical values. This tool was developed to recognize only relative changes in antibiotic spectrum and is not quantitative. A spectrum score of piperacillin/tazobactam of 42.3 could not be construed as 3 times as broad as that of vancomycin at 13 . Thus, we did not perform statistical analysis of the magnitude of changes because such analysis would be inconsistent with the intended purpose of the spectrum score method. Additionally, while this method demonstrated reliable classification of appropriate deescalation and escalation in previous studies, a case-by-case review determining appropriateness of antibiotic changes was not performed.

\section{Clinical Response}

This quality improvement study was initiated to determine whether positive blood culture results actually affect clinical management at MVAMC. The answer seems to be yes, with blood culture results altering antibiotic administration in about $60 \%$ of cases with the predominant change being deescalation. This overall rate of deescalation is toward the higher end of previously documented rates and coincides with the upper bound of the clinically advised deescalation rate described by Heenen and colleagues. ${ }^{38}$

As noted, the spectrum score is not quantitative. Still, one may be able to contend that the values may provide some insight into the magnitude of the changes in antibiotic selection. Deescalations were on average much larger changes in spectrum than escalations. 
The larger magnitude of deescalations reflects that when already starting with a very broad spectrum of coverage, it is much easier to get narrower than even broader. Stated another way, when starting therapy using piperacillin/ tazobactam at a spectrum score of 42.3 on a 60-point scale, there is much more room for deescalation to 0 than escalation to 60 . Additionally, escalations were more likely with much smaller of a spectrum change due to accurate empirical judgment of the suspected pathogens with new findings only necessitating a minor expansion of the spectrum of coverage.

Another finding within this investigation was the statistically significantly shorter response mean (SD) time when deescalating in response to pathogen identification $(8[7.3] \mathrm{h})$ than to sensitivity profile $(10.4[7] \mathrm{h})$. Overall when deescalating, the time of each individual response to antibiotic changes was highly irregular. There was no noticeable time point where a change was more likely to occur within the 24 hours after notification of a culture result. This erratic distribution further exemplifies the complexity of deescalation as it underscores the unique nature of each case. The timing of the dosage of previous antibiotics, the health status of the patient, and the individual physician attitudes about the progression and severity of the infection all likely played into this distribution.

Due to the lack of a regular or even skewed distribution, a Wilcoxon nonparametric rank sum test was performed $(P=.049)$. Although this result was statistically significant, the 2.5-hour time difference is likely clinically irrelevant as both times represent fairly prompt physician responsiveness. ${ }^{50}$ Nonetheless, it suggests that it was more important to rapidly escalate the breadth of coverage for a patient with a positive blood culture than to deescalate as identified pathogens may have been left untreated with the prescribed antibiotic.

\section{Future Study}

Similar studies designed using the spectrum score methodology would allow for more meaningful interinstitutional comparison of antibiotic administration through the use of a unified definition of deescalation and esca- lation. Comparison of deescalation and escalation rates between hospital systems with similar patient populations with and without prompt infectious disease review and phone notification of blood culture results could further verify the value of such a protocol. It could also help determine which empiric antibiotics may be most effective in individual patient morbidity and mortality outcomes, length of stay, costs, and the development of antibiotic resistance. Chou and colleagues found that only 49 of 130 responding VA facilities had antimicrobial stewardship teams in place with even fewer (29) having a formal policy to establish an antimicrobial stewardship program. ${ }^{11}$ This significant variation in the practices of VA facilities across the nation underscores the benefit to be gained from implementation of value-added protocols such as daily infectious disease case monitoring and microbiology laboratory phone notification of positive blood culture results as it occurs at MVAMC.

They also noted that systems of patientlevel antibiotic review, and the presence of at least one full-time infectious disease physician were both associated with a statistically significant decrease in the use of antimicrobials, corroborating the results of this analysis. ${ }^{11}$ Adapting the current system of infectious disease specialist review of positive blood culture results to use remote monitoring through the EHR could help to defer some of the cost of needing an inhouse specialist while retaining the benefit of the oversite.

Another option for study would be a before and after design to determine whether the program of infectious disease specialist review led to increased use of deescalation strategies similar to studies investigating the efficacy of antimicrobial subcommittee implementation. ${ }^{13,20,23,24,26}$

\section{CONCLUSIONS}

This analysis of empiric antibiotic use at the MVAMC indicates promising rates of deescalation. The results indicate that the medical service may be right and that positive blood culture results appear to affect clinical decision making in an appropriate and timely fashion. The VA is the largest health care organization in the US. Thus, identifying and propagating effective stewardship practices 
on a widespread basis can have a significant effect on the public health of the nation.

These data suggest that the program implemented at the MVAMC of phone notification to the primary care team along with daily infectious disease staff monitoring of blood culture information should be widely adopted at sister institutions using either inhouse or remote specialist review.

\section{Author disclosures}

The authors report no actual or potential conflicts of interest with regard to this article.

\section{Disclaimer}

The opinions expressed herein are those of the authors and do not necessarily reflect those of Federal Practitioner, Frontline Medical Communications Inc., the US Government, or any of its agencies. This article may discuss unlabeled or investigational use of certain drugs. Please review the complete prescribing information for specific drugs or drug combinations-including indications, contraindications, warnings, and adverse effectsbefore administering pharmacologic therapy to patients.

\section{References}

1. US Department of Veterans Affairs, Veterans Health Administration-About VHA. Updated January 22, 2021. Accessed February 19, 2021. https://www.va.gov/health /aboutvha.asp.

2. Masterton RG. Antibiotic de-escalation. Crit Care Clin. 2011;27(1):149-162. doi:10.1016/j.ccc.2010.09.009

3. Garnacho-Montero J, Gutiérrez-Pizarraya A, EscorescaOrtega $A$, et al. De-escalation of empirical therapy is associated with lower mortality in patients with severe sepsis and septic shock. Intensive Care Med. 2014;40(1):32-40. doi:10.1007/s00134-013-3077-7

4. Rhodes A, Evans LE, Alhazzani W, et al. Surviving sepsis campaign: international guidelines for management of sepsis and septic shock: 2016. Intensive Care Med. 2017;43(3):304-377. doi:10.1007/s00134-017-4683-6

5. Srinivasan A, Song $X$, Richards A, Sinkowitz-Cochran $R$, Cardo D, Rand C. A survey of knowledge, attitudes, and beliefs of house staff physicians from various specialties concerning antimicrobial use and resistance. Arch Intern Med. 2004;164(13):1451-1456. doi:10.1001/archinte.164.13.1451

6. Stach LM, Hedican EB, Herigon JC, Jackson MA, Newland JG. Clinicians' attitudes towards an antimicrobial stewardship program at a children's hospital. J Pediatric Infect Dis Soc. 2012;1(3):190-197. doi:10.1093/jpids/pis045

7. Salsgiver E, Bernstein D, Simon MS, et al. Knowledge, attitudes, and practices regarding antimicrobial use and stewardship among prescribers at acute-care hospitals. Infect Control Hosp Epidemiol. 2018;39(3):316-322. doi:10.1017/ice.2017.317

8. Bamgbola O. Review of vancomycin-induced renal toxicity: an update. Ther Adv Endocrinol Metab. 2016;7(3):136-147. doi:10.1177/2042018816638223

9. Kunni CM, Finland M. Restrictions imposed on antibiotic therapy by renal failure. Arch Intern Med. 1959;104:10301050. doi:10.1001/archinte.1959.00270120186021

10. Sartelli M, Catena F, Abu-Zidan FM, et al. Management of intra-abdominal infections: recommendations by the WSES 2016 consensus conference. World J Emerg Surg. 2017;12:22. Published 2017 May 4. doi:10.1186/s13017-017-0132-7

11. Chou AF, Graber CJ, Jones M, et al. Characteristics of antimicrobial stewardship programs at Veterans Affairs hospitals: results of a nationwide survey. Infect Control Hosp
Epidemiol. 2016;37(6):647-654. doi:10.1017/ice.2016.26

12. Giantsou E, Liratzopoulos N, Efraimidou E, et al. De-escalation therapy rates are significantly higher by bronchoalveolar lavage than by tracheal aspirate. Intensive Care Med. 2007;33(9):1533-1540. doi:10.1007/s00134-007-0619-x

13. Malani AN, Richards PG, Kapila S, Otto MH, Czerwinski J, Singal B. Clinical and economic outcomes from a community hospital's antimicrobial stewardship program. Am J Infect Control. 2013;41(2):145-148. doi:10.1016/j.ajic.2012.02.021

14. Souza-Oliveira AC, Cunha TM, Passos LB da S, Lopes GC, Gomes FA, Röder DVD de B. Ventilator-associated pneumonia: the influence of bacterial resistance, prescription errors, and de-escalation of antimicrobial therapy on mortality rates. Brazilian J Infect Dis. 2016;20(5):437-443. doi:10.1016/j.bjid.2016.06.006

15. Kim JW, Chung J, Choi SH, et al. Early use of imipenem/ cilastatin and vancomycin followed by de-escalation versus conventional antimicrobials without de-escalation for patients with hospital-acquired pneumonia in a medical ICU: a randomized clinical trial. Crit Care. 2012;16(1):R28. Published 2012 Feb 15. doi:10.1186/cc11197

16. Leone M, Bechis C, Baumstarck K, et al. De-escalation versus continuation of empirical antimicrobial treatment in severe sepsis: a multicenter non-blinded randomized noninferiority trial [published correction appears in Intensive Care Med. 2014 Nov;40(11):1794]. Intensive Care Med. 2014;40(10):1399-1408. doi:10.1007/s00134-014-3411-8

17. Gonzalez L, Cravoisy A, Barraud D, et al. Factors influencing the implementation of antibiotic de-escalation and impact of this strategy in critically ill patients. Crit Care. 2013;17(4):R140. Published 2013 Jul 12. doi: $10.1186 /$ cc12819

18. Safdar N, Handelsman J, Maki DG. Does combination antimicrobial therapy reduce mortality in Gram-negative bacteraemia? A meta-analysis. Lancet Infect Dis. 2004;4(8):519-527. doi:10.1016/S1473-3099(04)01108-9

19. Peña C, Suarez C, Ocampo-Sosa A, et al. Effect of adequate single-drug vs combination antimicrobial therapy on mortality in Pseudomonas aeruginosa bloodstream infections: a post hoc analysis of a prospective cohort. Clin Infect Dis. 2013;57(2):208-216. doi:10.1093/cid/cit223

20. Campion M, Scully G. Antibiotic Use in the Intensive Care Unit: Optimization and De-Escalation. J Intensive Care Med. 2018;33(12):647-655. doi:10.1177/0885066618762747

21. Mokart D, Slehofer G, Lambert J, et al. De-escalation of antimicrobial treatment in neutropenic patients with severe sepsis: results from an observational study. Intensive Care Med. 2014;40(1):41-49. doi:10.1007/s00134-013-3148-9

22. Li H, Yang $\mathrm{CH}$, Huang LO, et al. Antibiotics de-escalation in the treatment of ventilator-associated pneumonia in trauma patients: a retrospective study on propensity score matching method. Chin Med J (Engl). 2018;131(10):1151-1157. doi:10.4103/0366-6999.231529

23. Lindsay PJ, Rohailla S, Taggart LR, et al. Antimicrobial stewardship and intensive care unit mortality: a systematic review. Clin Infect Dis. 2019;68(5):748-756. doi:10.1093/cid/ciy550

24. Perez KK, Olsen RJ, Musick WL, et al. Integrating rapid diagnostics and antimicrobial stewardship improves outcomes in patients with antibiotic-resistant Gramnegative bacteremia. J Infect. 2014;69(3):216-225 doi:10.1016/j.jinf.2014.05.005

25. Ikai H, Morimoto T, Shimbo T, Imanaka Y, Koike K. Impact of postgraduate education on physician practice for community-acquired pneumonia. J Eval Clin Pract. 2012;18(2):389-395. doi:10.1111/j.1365-2753.2010.01594.x

26. Ruiz J, Ramirez P, Gordon M, et al. Antimicrobial stewardship programme in critical care medicine: A prospective interventional study. Med Intensiva. 2018;42(5):266-273. doi:10.1016/j.medin.2017.07.002

27. Berild D, Mohseni A, Diep LM, Jensenius M, Ringertz SH. Adjustment of antibiotic treatment according to the results of blood cultures leads to decreased antibiotic use 
and costs. J Antimicrob Chemother. 2006;57(2):326-330. doi:10.1093/jac/dki463

28. Davey P, Brown E, Charani E, et al. Interventions to improve antibiotic prescribing practices for hospital inpatients. Cochrane Database Syst Rev. 2013;(4):CD003543. Published 2013 Apr 30. doi:10.1002/14651858.CD003543.pub3

29. Centers for Disease Control and Prevention. Antibiotic resistance threats in the United States, 2019. Revised December 2019. Accessed March 2, 2021. https://www.cdc .gov/drugresistance/pdf/threats-report/2019-ar-threats -report-508.pdf

30. O'Neill J. Antimicrobial resistance: tackling a crisis for the health and wealth of nations. Published December 2014. Accessed February 19, 2021. https://amr-review.org /sites/default/files/AMR\%20Review\%20Paper\%20-\%20 Tackling $\% 20$ a $\% 20$ crisis $\% 20$ for $\% 20$ the $\% 20$ health $\% 20$ and\%20wealth\%20of\%20nations_1.pdf

31. Rhodes A, Evans LE, Alhazzani W, et al. Surviving sepsis campaign: international guidelines for management of sepsis and septic shock: 2016. Intensive Care Med. 2017;43(3):304-377. doi:10.1007/s00134-017-4683-6

32. De Waele JJ, Akova M, Antonelli M, et al. Antimicrobial resistance and antibiotic stewardship programs in the ICU: insistence and persistence in the fight against resistance. A position statement from ESICM/ESCMID/WAAAR round table on multi-drug resistance. Intensive Care Med. 2018;44(2):189-196. doi:10.1007/s00134-017-5036-1

33. Madaras-Kelly K, Jones M, Remington R, Hill N, Huttner B, Samore M. Development of an antibiotic spectrum score based on veterans affairs culture and susceptibility data for the purpose of measuring antibiotic de-escalation: a modified Delphi approach. Infect Control Hosp Epidemiol. 2014;35(9):1103-1113. doi:10.1086/677633

34. Tabah A, Cotta MO, Garnacho-Montero J, et al. A systematic review of the definitions, determinants, and clinical outcomes of antimicrobial de-escalation in the intensive care unit. Clin Infect Dis. 2016;62(8):1009-1017. doi:10.1093/cid/civ1199

35. Primaxin IV. Prescribing information. Merck \& Co, Inc; 2001. Accessed February 23, 2021. https://www.merck. $\mathrm{com} /$ product/usa/pi_circulars/p/primaxin/primaxin iv_pi.pdf

36. Coccolini F, Trevisan M, Montori G, et al. Mortality rate and antibiotic resistance in complicated diverticulitis: report of 272 consecutive patients worldwide: a prospective cohort study. Surg Infect (Larchmt). 2017;18(6):716-721. doi:10.1089/sur.2016.283

37. Selva Olid A, Solà I, Barajas-Nava LA, Gianneo OD, Bonfill Cosp X, Lipsky BA. Systemic antibiotics for treating diabetic foot infections. Cochrane Database Syst Rev. 2015;(9):CD009061. Published 2015 Sep 4. doi:10.1002/14651858.CD009061.pub2

38. Heenen S, Jacobs F, Vincent JL. Antibiotic strategies in severe nosocomial sepsis: why do we not de-escalate more often?. Crit Care Med. 2012;40(5):1404-1409. doi:10.1097/CCM.0b013e3182416ecf

39. Morel J, Casoetto J, Jospé R, et al. De-escalation as part of a global strategy of empiric antibiotherapy management. $A$ retrospective study in a medico-surgical intensive care unit. Crit Care. 2010;14(6):R225. doi:10.1186/cc9373

40. Moraes RB, Guillén JA, Zabaleta WJ, Borges FK. Deescalation, adequacy of antibiotic therapy and culture positivity in septic patients: an observational study. Descalonamento, adequação antimicrobiana e positividade de culturas em pacientes sépticos: estudo observacional. Rev Bras Ter Intensiva. 2016;28(3):315-322. doi:10.5935/0103-507X.20160044

41. Khasawneh FA, Karim A, Mahmood T, et al. Antibiotic de-escalation in bacteremic urinary tract infections: potential opportunities and effect on outcome. Infection. 2014;42(5):829-834. doi:10.1007/s15010-014-0639-8

42. Alshareef $H$, Alfahad W, Albaadani A, Alyazid H, Talib RB. Impact of antibiotic de-escalation on hospitalized patients with urinary tract infections: A retrospective cohort single center study. J Infect Public Health. 2020;13(7):985-990. doi:10.1016/j.jph.2020.03.004

43. De Waele JJ, Schouten J, Beovic B, Tabah A, Leone M. Antimicrobial de-escalation as part of antimicrobial stewardship in intensive care: no simple answers to simple questions-a viewpoint of experts. Intensive Care Med. 2020;46(2):236-244. doi:10.1007/s00134-019-05871-z

44. Eachempati SR, Hydo LJ, Shou J, Barie PS. Does deescalation of antibiotic therapy for ventilator-associated pneumonia affect the likelihood of recurrent pneumonia or mortality in critically ill surgical patients?. J Trauma. 2009:66(5):1343-1348. doi:10.1097/TA.0b013e31819dca4e

45. Kollef MH, Morrow LE, Niederman MS, et al. Clinical characteristics and treatment patterns among patients with ventilator-associated pneumonia [published correction appears in Chest. $2006 \mathrm{Jul} ; 130(1): 308]$. Chest. 2006;129(5):1210-1218. doi:10.1378/chest.129.5.1210

46. Gerding DN, Johnson S, Peterson LR, Mulligan ME, Silva $\mathrm{J}$ Jr. Clostridium difficile-associated diarrhea and colitis. Infect Control Hosp Epidemiol. 1995;16(8):459-477. doi:10.1086/648363

47. Pépin J, Saheb N, Coulombe MA, et al. Emergence of fluoroquinolones as the predominant risk factor for Clostridium difficile-associated diarrhea: a cohort study during an epidemic in Quebec. Clin Infect Dis. 2005;41(9):1254-1260. doi:10.1086/496986

48. Seddon MM, Bookstaver PB, Justo JA, et al. Role of Early De-escalation of Antimicrobial Therapy on Risk of Clostridioides difficile Infection Following Enterobacteriaceae Bloodstream Infections. Clin Infect Dis. 2019;69(3):414420. doi:10.1093/cid/ciy863

49. Livorsi D, Comer A, Matthias MS, Perencevich EN, Bair MJ. Factors influencing antibiotic-prescribing decisions among inpatient physicians: a qualitative investigation. Infect Control Hosp Epidemiol. 2015;36(9):1065-1072. doi:10.1017/ice.2015.136

50. Liu P, Ohl C, Johnson J, Williamson J, Beardsley J, Luther $V$. Frequency of empiric antibiotic de-escalation in an acute care hospital with an established antimicrobial stewardship program. BMC Infect Dis. 2016;16(1):751. Published 2016 Dec 12. doi:10.1186/s12879-016-2080-3 